\begin{tabular}{|c|l|}
\hline Title & Transient deformation of a droplet near a microfluidic constriction : A quantitative analysis \\
\hline Author(s) & Tregouet, Corentin; Salez, Thomas; Monteux, Cecile; Reyssat, Mathilde \\
\hline Citation & $\begin{array}{l}\text { Physical Review Fluids, 3(5), 053603 } \\
\text { https://doi.org/10.1103/PhysRevFluids.3.053603 }\end{array}$ \\
\hline Issue Date & 2018.05-18 \\
\hline Doc URL & http://hdl.handle.net/2115/71061 \\
\hline Rights & @2018A American Physical Society \\
\hline Type & article \\
\hline File Information & PhysRevFluids.3.053603.pdf \\
\hline
\end{tabular}

Instructions for use 


\title{
Transient deformation of a droplet near a microfluidic constriction: A quantitative analysis
}

\author{
Corentin Trégouët, ${ }^{1,2}$ Thomas Salez, ${ }^{3,4, *}$ Cécile Monteux,,${ }^{1,4}$ and Mathilde Reyssat ${ }^{2, *}$ \\ ${ }^{1}$ UMR CNRS SIMM 7615, ESPCI Paris, PSL Research University, 75005 Paris, France \\ ${ }^{2}$ UMR CNRS Gulliver 7083, ESPCI Paris, PSL Research University, 75005 Paris, France \\ ${ }^{3}$ Université de Bordeaux, CNRS, LOMA, UMR 5798, F-33405 Talence, France \\ ${ }^{4}$ Global Station for Soft Matter, Global Institution for Collaborative Research and Education, \\ Hokkaido University, Sapporo, Japan
}

(Received 18 January 2018; published 18 May 2018)

\begin{abstract}
We report on experiments that consist of deforming a collection of monodisperse droplets produced by a microfluidic chip through a flow-focusing device. We show that a proper numerical modeling of the flow is necessary to access the stress applied by the latter on the droplet along its trajectory through the chip. This crucial step enables the full integration of the differential equation governing the dynamical deformation, and consequently the robust measurement of the interfacial tension by fitting the experiments with the calculated deformation. Our study thus demonstrates the feasibility of quantitative in situ rheology in microfluidic flows involving, e.g., droplets, capsules, or cells.
\end{abstract}

DOI: 10.1103/PhysRevFluids.3.053603

\section{INTRODUCTION}

Droplet-based microfluidics enables the fragmentation of compounds (chemicals, cells, etc.) into nanoliters. Such microdroplets can be further protected by a thin elastic shell realized through several microencapsulation techniques. The inner phase of such capsules can then be easily transported and delivered into specific places [1-5]. At the microscale, the deformation of droplets or capsules is controlled by a balance between the driving forces which deform the objects (shear, elongation, etc.) and the restoring ones which result from their interfacial properties: the interfacial tension in the case of droplets or the interfacial elasticity in the case of capsules, as well as the bulk and interfacial viscosities. Measuring the interfacial properties in situ in a microfluidic channel is particularly relevant as it can give insights into the dynamical composition, stability, and rheology of such interfaces (including encapsulation membranes), which is crucial to understand and optimize their behavior.

For simple droplets, different approaches have been investigated in the past to measure interfacial tension by shear deformation. The first quantitative experiments of droplet deformation under viscous stress were performed by Taylor in 1934 [6], using the four-roller apparatus. Therein, the fluid arrives in a two-dimensional (2D) cross-shaped location from two opposite sides and leaves on the two orthogonal others. The droplet is thus sheared and deformed. The steady shape in that case results from a balance between the driving viscous stress and the restoring interfacial tension. Consequently, the knowledge of the viscous shear stress at the interface and the measurement of the deformation lead to a measurement of the interfacial tension.

Lee et al. [7] and Deschamps et al. [8] miniaturized Taylor's experiment on a microfluidic chip to measure interfacial properties of microdroplets and vesicles. More recently, Xie et al. [9] used

\footnotetext{
*Corresponding authors: thomas.salez@u-bordeaux.fr; mathilde.reyssat@espci.fr
} 
similar geometry to characterize the interfacial rheological properties of microcapsules. Hudson et al. further integrated this device into a microfluidic channel by using a convergent and then divergent channel profile $[10,11]$. That device generates a gradient of velocity in the main direction of the flow, leading to shear stress and thus droplet deformation. This in-flow process then allows researchers to perform measurements on a large number of droplets, and hence enables a statistical treatment of the data, which enhances the accuracy of the results. The previous setup has been further adapted by Brosseau et al. [12] in order to deform capsules, which require a stronger stress. This last geometry is composed of an alternating succession of narrow channels, in which the capsules are confined by the walls, and wide chambers, where they only interact with the viscous flow. The transition between two parts is sharp in the latter geometry.

Hudson et al. have shown that the droplets in the in-flow device are in a transient regime [10,11]. In specific conditions (geometry, viscosity contrast), the authors have succeeded in extracting the interfacial tension by dynamically analyzing the shape of drops but their approach relies on a derivative of the experimental data, which can induce large errors. However, the evolution of such nonstationary shapes has been described theoretically by Barthes-Biesel et al. [13], which could in principle be used to get interfacial tension in any configuration. Up to now, the precise evolution of the shape of a droplet in any geometry and its fitting to theoretical predictions has not been performed.

In the present study, we perform experiments that consist of deforming a collection of monodisperse droplets produced by a microfluidic chip through a flow-focusing device. We show that a proper numerical modeling of the flow is necessary to access the stress applied by the latter on the droplet along its trajectory through the chip. This crucial step enables the full integration of the differential equation governing the dynamical deformation, and consequently the robust measurement of the interfacial tension by fitting the experiments with the calculated deformation.

\section{MATERIAL AND METHODS}

\section{A. Materials}

The droplets consist of mineral oil (Sigma Aldrich, mineral oil rotational viscosity standard of $29.04 \mathrm{mPa} s$ at $25.00^{\circ} \mathrm{C}$ ) flowing in a polymer solution [poly(methacrylic acid), PMAA, provided by Polysciences] at $1 \mathrm{wt} \%$ adjusted at $\mathrm{pH} 3$. The droplets are produced and characterized in the same polymer solution (PMAA $1 \mathrm{wt} \%, \mathrm{pH} 3$ ). They are produced in microfluidics using a standard flowfocusing device made of poly(dimethyl siloxane) (PDMS). Such a setup provides a very monodisperse collection of droplets ranging from 50 to $70 \mu \mathrm{m}$ in diameter. The characterization chips are realized with a photosensitive adhesive provided by Norland (NOA 81). This microfabrication technique allows one to build nondeformable channels confined between two glass surfaces, which is able to sustain large flow rates without being deformed [14]. The inlet is connected to the droplet-production chip by a silicon tubing (Tygon) of inner diameter $800 \mu \mathrm{m}$, coated with a solution of bovine serum albumine (BSA) provided by Sigma Aldrich, to prevent adhesion of the droplets inside the tubing (incubation at $1 \mathrm{wt} \%$ solution during $12 \mathrm{~h}$ at room temperature). The outlet is connected to a Peek tubing of inner diameter $125 \mu \mathrm{m}$.

\section{B. Methods}

\section{Observation of the deformation}

The characterization chip consists of a sharp transition between a narrow channel (of width $W=40 \mu \mathrm{m}$ ) and a wide chamber (of width $3 W$ ), similar to the chip used by Polenz et al. [15]. Such a geometry imposes a divergent flow near the entrance of the wide chamber, as illustrated by fluorescent tracers in Fig. 1(a) (picture realized in a channel of width $W=80 \mu \mathrm{m}$ ). The height in the $z$ direction is constant and equals $100 \mu \mathrm{m}$, which prevents the droplets from being confined in the $z$ direction.

The divergent flow at the entrance of the wide chamber generates a viscous stress on the droplets, which tends to elongate them perpendicularly to the flow direction. We note that the convergent flow 


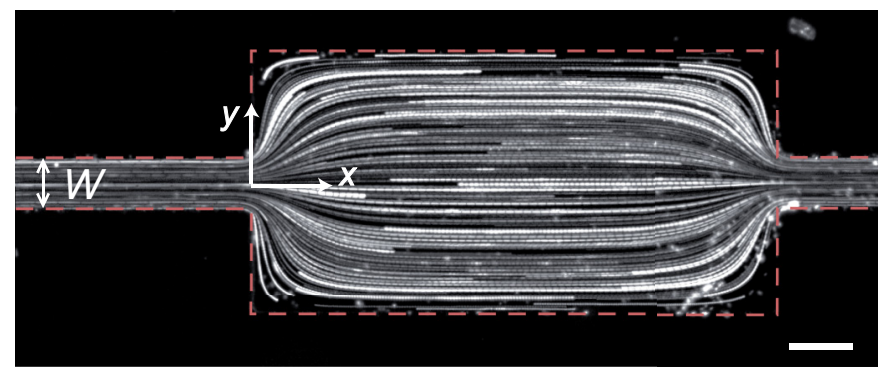

(a)

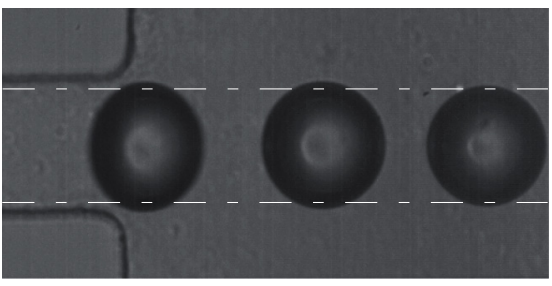

(b)

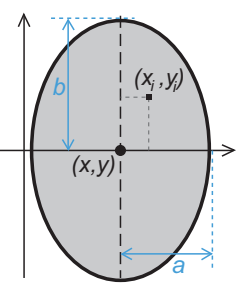

(c)

FIG. 1. (a) Characterization chip. Streamlines (from left to right) are visible due to fluorescent tracers and show the divergent flow at the entrance of the wide chamber. Scale bar is $100 \mu \mathrm{m}$. (b) Droplet entering the wide chamber. The droplet is first elongated along $y$ and then relaxes toward a spherical shape (stack of 3 images). The deformation of a few percent is barely visible by eye. (c) Schematics showing the parameters $a$ and $b$ describing the droplet shape. This configuration corresponds to a positive deformation of the droplet.

at the exit of the wide chamber could also be used to deform the droplets. However, the narrow channel better aligns the droplets with the center of the wide chamber at its entrance than at its exit-especially when the droplets are laterally confined in the narrow channel, which is the case in our experiments. As a consequence, for more reproducible results, observations and measurements are performed only at the entrance of the wide chamber. The flow is controlled by a pressure controller (Fluigent).

Observation is performed along the $z$ direction through a Leica inverted microscope with a $10 \times$ objective. A Photron Fastcam-SA camera mounted on the microscope allows us to record the droplet deformation at high acquisition frequency (typically $10000 \mathrm{fps}$ ). Pictures similar to the ones presented in Fig. 1(b) are recorded every $0.1 \mathrm{~ms}$ during $2 \mathrm{~s}$. The different steps of the image processing needed to get the deformation measurement as a function of position in the wide chamber are detailed in the Appendix.

\section{Governing equation}

To relate the measured deformation to interfacial tension, Taylor developed a theoretical model [6], which was generalized by Barthes-Biesel et al. [13,16] and summarized by Rallison [17]. This model has been used by Hudson et al.[10] in order to extract interfacial tension values.

The model of Taylor [6] considers a droplet of viscosity $\eta_{\text {droplet }}$ placed in a fluid medium of viscosity $\eta_{\text {medium }}$. The radius of the droplet at rest is $r$, and the interfacial tension with the surrounding fluid is $\gamma$. In Taylor's model, the latter fluid undergoes a hyperbolic flow in the $(x, y)$ plane with a velocity field that reads $\underline{u}=\left(u_{x}, u_{y}\right)=C(x,-y)$, where $C$ is a constant fixed by the flux. The extension rate $\dot{\epsilon}$ along $x$ is thus related to the gradient of velocity of the fluid along $x$, through $\dot{\epsilon}=\frac{\mathrm{d} u_{x}}{\mathrm{~d} x}=C$. In this configuration, $\dot{\epsilon}$ does not depend on the position, and the steady deformation $D_{\text {steady }_{\text {Taylor }}}$ (see 
definition of the deformation $D$ in Appendix) of the droplet is directly proportional to $\dot{\epsilon}$ [6], as

$$
D_{\text {steady }_{\text {Taylor }}}=\frac{19 \lambda+16}{16 \lambda+16} \frac{\eta_{\text {medium }} r}{\gamma} \dot{\epsilon},
$$

where $\lambda=\frac{\eta_{\text {droplet }}}{\eta_{\text {medium }}}$ is the viscosity ratio between the droplet and the fluid medium.

When the flow is less regular than the hyperbolic flow described by Taylor, Barthes-Biesel et al. [13] state that for moderated shear stress and hence deformation, the flow in the vicinity of the droplet can be approximated to the first order in deformation and described by a deformation-rate tensor independent of position. In these conditions, the steady deformation must be calculated with the eigenvalues $e_{1}$ and $e_{2}$ of the deformation-rate tensor [see definition in Eq. (8)], through

$$
D_{\text {steady }}=\frac{19 \lambda+16}{16 \lambda+16} \frac{\eta_{\text {medium }} r}{\gamma}\left(e_{1}-e_{2}\right) \text {. }
$$

Furthermore, Barthes-Biesel et al. [13] describe the transient regime as a first-order relaxation toward the steady state of Eq. (2). In this framework, the deformation $D(t)$ satisfies

$$
\frac{\mathrm{d} D}{\mathrm{~d} t}=\frac{1}{\tau_{\mathrm{ca}}}\left(D_{\text {steady }}-D\right)
$$

with a relaxation time $\tau_{\mathrm{ca}}$ defined as

$$
\tau_{\mathrm{ca}}=\frac{2}{5}(2 \lambda+3) \frac{19 \lambda+16}{16 \lambda+16} \frac{\eta_{\text {medium }} r}{\gamma} .
$$

The droplet-medium interfacial tension $\gamma$ can thus be obtained by fitting the experimental deformation $D(t)$ of the droplet using Eqs. (3) and (4). The only requirement is to know precisely the positiondependent steady deformation $D_{\text {steady }}$ of the droplet in the considered geometry.

\section{Calculation of the steady deformation}

Our geometry is described in Sec. II B 1. In the absence of any symmetry, and including further a moving droplet, it is not straightforward to know precisely the viscous stress exerted by the surrounding fluid on the droplet. Performing finite-element numerical simulations is a robust way to access this information, in order to further calculate the position-dependent steady deformation $D_{\text {steady }}$ of the droplets. The flow field and the associated velocity gradient are extracted from such simulations (using the software COMSOL MULTIPHYSICS), and analyzed to calculate the steady deformation of the droplets.

Framework of the simulations. The simulated geometry consists of a fixed hard sphere placed at the position $(x, 0,0)$ near the entrance of a wide chamber three times larger (in $y)$ than the incoming narrow channel, but with identical height (in $z$ ). The hard-sphere idealization is justified by the tiny experimental deformations that alter the flow only marginally. Calibration experiments are performed with tracers (of diameter $5 \mu \mathrm{m}$ ) in order to know precisely the relative speed between the medium and the droplet. It appears that at a distance $x=150 \mu \mathrm{m}$, the droplet is nearly spherical and its velocity is similar to the medium speed. Consequently, in each experiment, we determine the rest diameter of the droplet in the far field (where the drop is not deformed), and its velocity in this region gives access to the flow velocity and thus the flow rate of the surrounding fluid everywhere in the channel. Therefore, in each simulation, the droplet diameter and the flow rate can be set to mimic precisely a given experiment. To analyze a given experiment, simulations are made for different positions of the droplet (typically 10 points between $x=20 \mu \mathrm{m}$ and $x=120 \mu \mathrm{m}$ ). In such simulations, the droplet does not move by design, as we only consider steady states in this part of the modeling.

Boundary conditions. We assume a no-slip condition at the walls, which implies $\underline{u}=\underline{0}$ there since the walls are not permeable and/or deformable. That assumption is valid, since the typical length scales of our microfluidic device are much larger than the slip length in a waterlike solution near a glassy wall, as the latter slip length seldom exceeds $100 \mathrm{~nm}$, according to Lauga et al. [18]. 
In contrast, the boundary condition at the droplet surface is not straightforward. First, we assume that there is no slip between the medium and the droplet and that there is no velocity in the droplet fluid at the droplet surface. These assumptions are motivated by our high viscosity ratio $\lambda=30$, together with the slow dynamics of the polymers at the interface. Eggleton et al. [19] showed that a no-slip boundary condition at the interface is obtained when the viscous drag which tends to cause polymer concentration gradients is compensated by a Marangoni flow in the opposite direction, resulting in zero tangential velocity. According to Ref. [19], even very small surface tension gradients can compensate viscous drag. Moreover, the Peclet number which represents the ratio of convective motion over diffusive motion is large in our experiment, $\mathrm{Pe}=\frac{\|\underline{u}\| R}{D_{\text {diffusion }}} \sim 10^{5}$, where $D_{\text {diffusion }} \sim 10^{-11} \mathrm{~m} / \mathrm{s}^{2}$ is the diffusion constant of a polymer chain in the solvent. As the polymers cannot rearrange by diffusion at the interface in such a short experimental timescale (around $1 \mathrm{~ms}$ ), any small surface tension gradient will be maintained and the no-slip condition justified. Second, we neglect the deformability of the droplet at leading order, as already explained. Third, the hard sphere is fixed in the simulations, whereas we need to determine the stress on a moving droplet. However, because inertia is negligible (Reynolds number of $\sim 0.1$ ), the fluid velocity field at time $t$ only depends on the boundary condition at the same time $t$. Thus, for each position $x$ of the fixed model hard sphere in the channel, the proper flow boundary condition is an effective slip boundary condition with a nonzero medium velocity $\underline{u(\Sigma)}$ on the sphere-medium interface $\Sigma$, matching the actual experimental droplet velocity:

$$
\underline{u(\Sigma)}=\frac{\mathrm{d} x}{\mathrm{~d} t} \underline{e}_{x}
$$

where $\underline{e}_{x}$ is the unit vector along the $x$ axis.

Analysis of the simulation data. The analysis of the raw simulation data is performed with MATLAB. Simulations provide as an output the velocity gradient for every point $M$ :

$$
\underline{\underline{\operatorname{grad}}} \underline{\underline{u}}=\left[\begin{array}{ccc}
u_{x x} & u_{x y} & u_{x z} \\
u_{y x} & u_{y y} & u_{y z} \\
u_{z x} & u_{z y} & u_{z z}
\end{array}\right],
$$

where $u_{x y}$ is the derivative of the $x$ component of the velocity field $\underline{u}$ with respect to the $y$ coordinate. For every location $x$ of the hard-sphere center, an arbitrary neighboring layer around the hard sphere is defined, with a thickness equal to 5\% of the sphere diameter. All the analysis is performed in this layer and we checked that a variation of the layer thickness does not influence our results. The deformation-rate tensor $\underline{\underline{d}}$ is defined as the symmetric component of the previous velocity gradient:

$$
\underline{\underline{d}}=\frac{1}{2}\left[\underline{\underline{\operatorname{grad}}} \underline{\underline{u}}+(\underline{\underline{\operatorname{grad}}} \underline{\underline{u}})^{\top}\right]
$$

In our specific geometry, we can reasonably assume that the stress is mostly in the $(x, y)$ plane. Consequently, we in fact only consider the associated bidimensional block $\underline{\underline{d}}_{2 \mathrm{D}}$ of the deformationrate tensor, defined as follows:

$$
\underline{\underline{d}}_{2 \mathrm{D}}=\left[\begin{array}{cc}
u_{x x} & \frac{1}{2}\left(u_{x y}+u_{y x}\right) \\
\frac{1}{2}\left(u_{y x}+u_{x y}\right) & u_{y y}
\end{array}\right] .
$$

Diagonalization of $\underline{\underline{d}}_{2 \mathrm{D}}$ gives two eigenvalues $e_{1}(M)$ and $e_{2}(M)$ for each point $M$. Both eigenvalues $e_{1}(M)$ and $e_{2}(M)$ are then averaged for every $M$ in the boundary layer. We would like to note that in the description by Barthes-Biesel et al. [13], a development of the flow in the vicinity of the droplet is considered and only the linear term is kept, where by construction the coefficient of the deformation rate tensor and hence the eigenvalues are constant. On the contrary, in our description, by diagonalizing locally, we get eigenvalues which depend on the position $M$ along the deformed object. We measure a relative standard deviation of the eigenvalues close to 1 , which quantifies the stress inhomogeneity observed in Fig. 3(a). By averaging these values for every $M$ in the boundary 
layer, we get rid of the fluctuations and recover the previous description. Those averaged values, $\left\langle e_{1}\right\rangle$ and $\left\langle e_{2}\right\rangle$, obtained for a given droplet position $x$, can finally be used in Eq. (2), in order to specify the position-dependent steady deformation $D_{\text {steady }}$ at stake.

\section{RESULTS AND DISCUSSION}

\section{A. Validation of the simulations}

As a preliminary check, our numerical procedure to extract the position-dependent steady deformation $D_{\text {steady }}$ is tested for a known geometry (different from ours) of the literature. Specifically, we consider the setup of Hudson et al. $[10,11,20]$. In their geometry, the transition between the narrow channel and the wide chamber is much smoother than ours. In addition, their droplet diameter is smaller than the width (and height) of the narrow channel. Note also that, all along this test, we use a modified viscosity ratio that actually matches the one of our experiments $(\lambda=30)$. As will be clear below, this specific choice enables the obtention of a second piece of information from our test (besides checking our numerical procedure): It allows us to demonstrate that Hudson et al.'s simplified approach is not valid for our experiments.

The position-dependent steady deformation calculated by Hudson et al. [10] is based on Taylor's equation (1) together with the following assumption:

$$
\dot{\epsilon}=\frac{\mathrm{d} v_{\text {droplet }}}{\mathrm{d} x},
$$

where $v_{\text {droplet }}$ is the droplet velocity along the $x$ axis. Indeed, these authors assume that the droplet only acts as a tracer, i.e., it has the exact same speed as the surrounding fluid and does not modify the flow. This assumption is motivated by both the small size of their droplets compared to the length scales of their microfluidic setup and their low viscosity ratio $(\lambda<1)$.

We perform two simulations for Hudson et al.'s geometry: one for the case of a tracer, i.e., without any actual droplet (in practice we calculate the stress on a phantom sphere), and one with a droplet (in practice we calculate the stress on a hard sphere with no slip, see Sec. II B 3). Figure 2 represents the comparison between the result of each of these two simulations and the model proposed by Hudson et al. In the tracer case [Fig. 2(a)], there is a good agreement between our simulation and the model proposed by Hudson et al., without any adjustable parameter. This self-consistency check indicates that our procedure calculates correctly $D_{\text {steady }}$ for a tracer. In contrast, in the no-slip hard-sphere case [Fig. 2(b)], there is a clear discrepancy between our simulation and the model proposed by Hudson et al. Since we are confident in the mathematical validity of our code thanks to the self-consistency check above, we conclude that even in a nonconfined and smooth geometry such as the one of Hudson et al., a very viscous droplet ( $\lambda=30$ here) actually modifies largely the flow field around. The model proposed by Hudson et al. does not apply in our case, and one therefore needs to perform a numerical simulation to know precisely $D_{\text {steady }}$. It is interesting to note that Taylor [6] also observed a discrepancy between his experiments and theoretical predictions when the viscosity ratio $\lambda$ became significantly higher than 1 .

\section{B. Solving the governing equation}

As explained in Sec. II B 3, the finite-element simulations allow us to compute the deformation-rate tensor at the surface of the droplet as a function of the droplet position $x$ in the wide chamber, and thus the viscous stress it experiences along its trajectory [see Fig. 3(a)]. From the diagonalization of the deformation-rate tensor, one can precisely calculate $D_{\text {steady }}(x)$ using Eq. (2) [see Fig. 3(b)]. Finally, integrating Eq. (3) along the trajectory of a droplet advected by the flow with the first measured point as an initial condition gives the position-dependent theoretical deformation $D_{\text {th }}(x)$ for a given interfacial tension.

Figure 4(a) presents different predictions of $D_{\text {th }}(x)$, for the droplet-medium interfacial tension $\gamma$ ranging from 20 to $250 \mathrm{mN} / \mathrm{m}$. In all cases, the deformation is initially negative, then it rapidly 


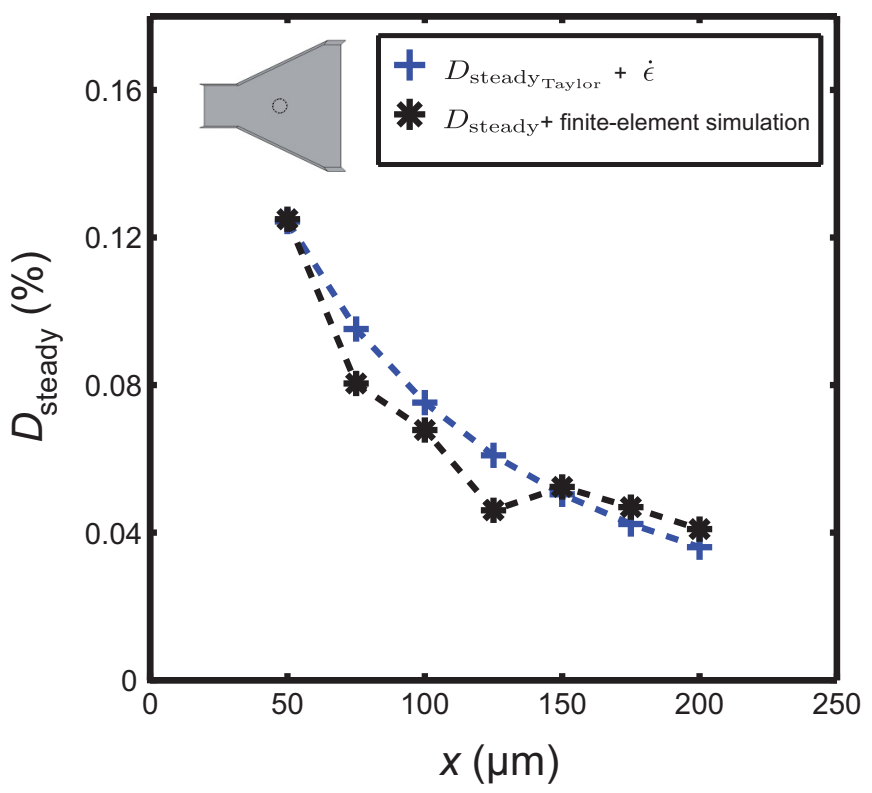

(a)

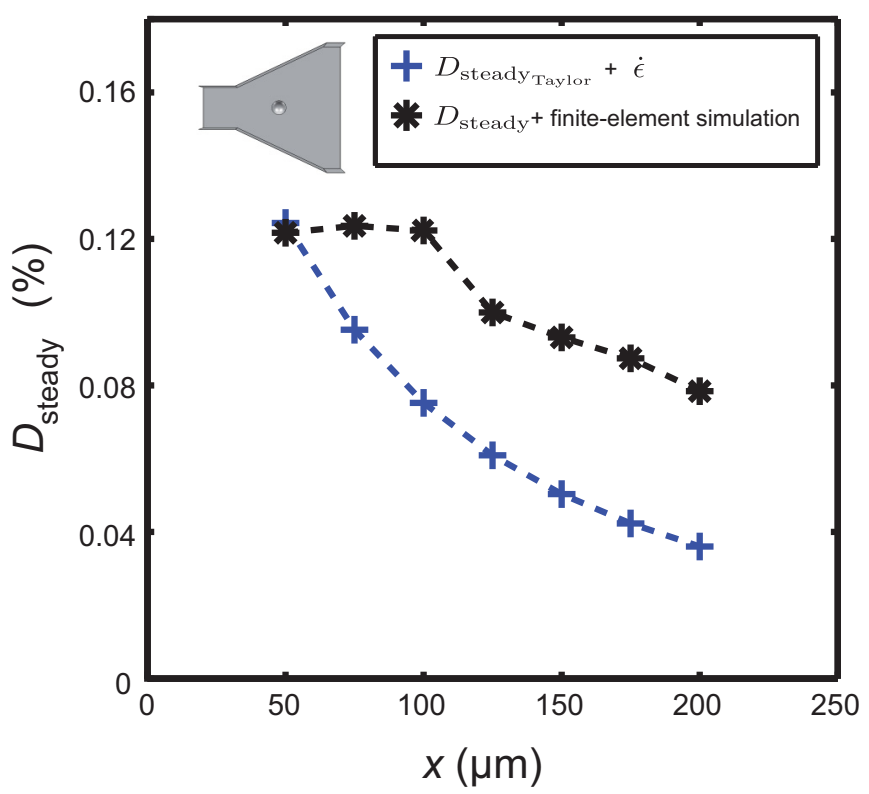

(b)

FIG. 2. (a) Steady deformation [see Eq. (2)] as a function of position, as obtained from a finite-element simulation (see Sec. II B 3) of an experiment in Hudson et al.'s geometry [10] (modified viscosity ratio $\lambda=30$ ). The droplet is replaced by a phantom sphere that does not disturb the flow. For comparison, we plot Eq. (1) using Eq. (9) $\left(D_{\text {steady Taylor }}+\dot{\epsilon}\right)$. (b) Same as panel (a), but when the droplet is actually as a no-slip hard-sphere obstacle. For comparison, we plot Eq. (1) using Eq. (9) $\left(D_{\text {steady }}\right.$ Taylor $\left.+\dot{\epsilon}\right)$. 


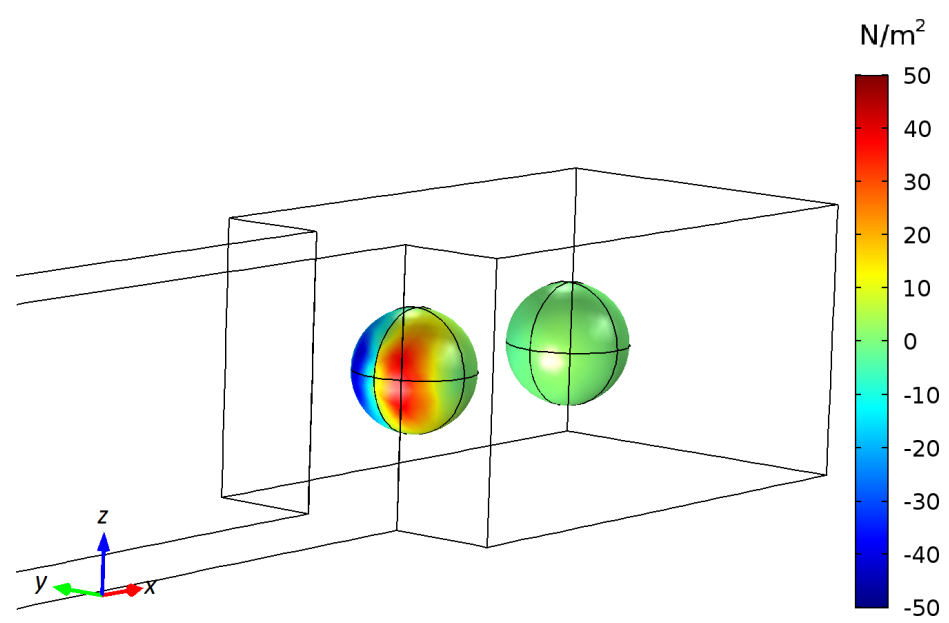

(a)

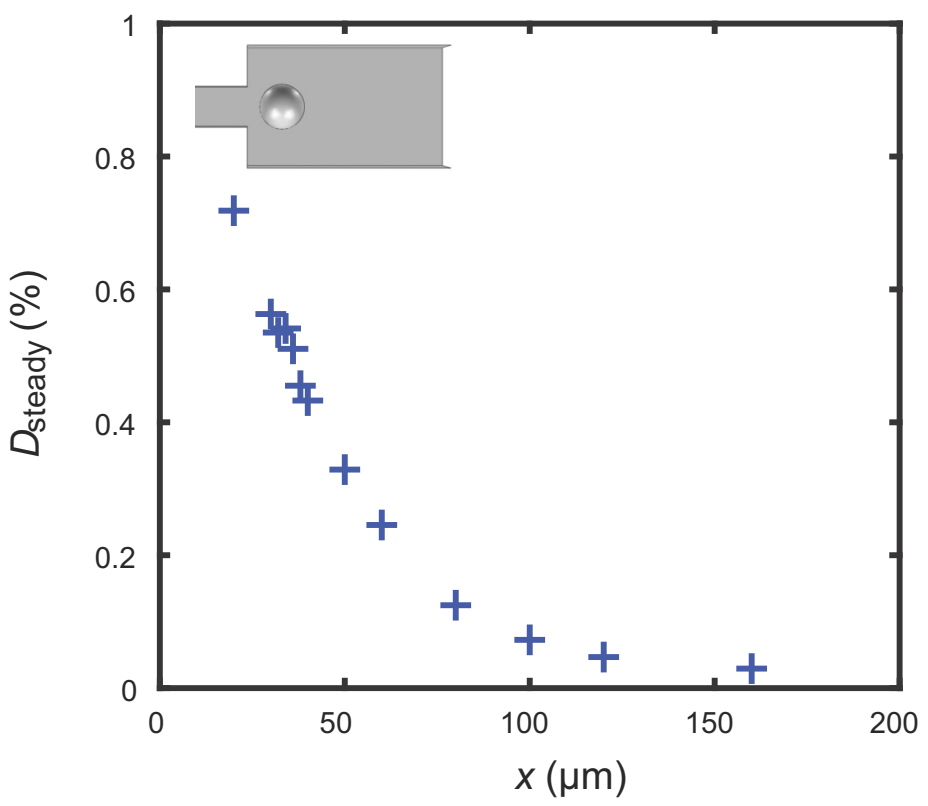

(b)

FIG. 3. (a) $y$ component of the steady viscous stress at the surface of the droplet, at both the entrance and in the middle of the wide chamber, as obtained from finite-element simulations (see Sec. IIB 3) of an experiment in our geometry (viscosity ratio $\lambda=30$ ). The droplet is modeled as a no-slip hard-sphere obstacle. (b) Resulting steady deformation [see Eq. (2)] as a function of position. The steady deformation is presented here for a surface tension chosen equal to $72 \mathrm{mN} / \mathrm{m}$.

becomes positive and reaches a maximum value, before slowly relaxing toward zero. We also observe that an increase in interfacial tension leads to a smaller value of the maximum deformation and to a faster overall dynamics. Therefore, thanks to the large influence of the interfacial tension on the droplet deformation in the transient regime, fitting the experimental data with those predictions enables a precise measurement of the interfacial tension. 


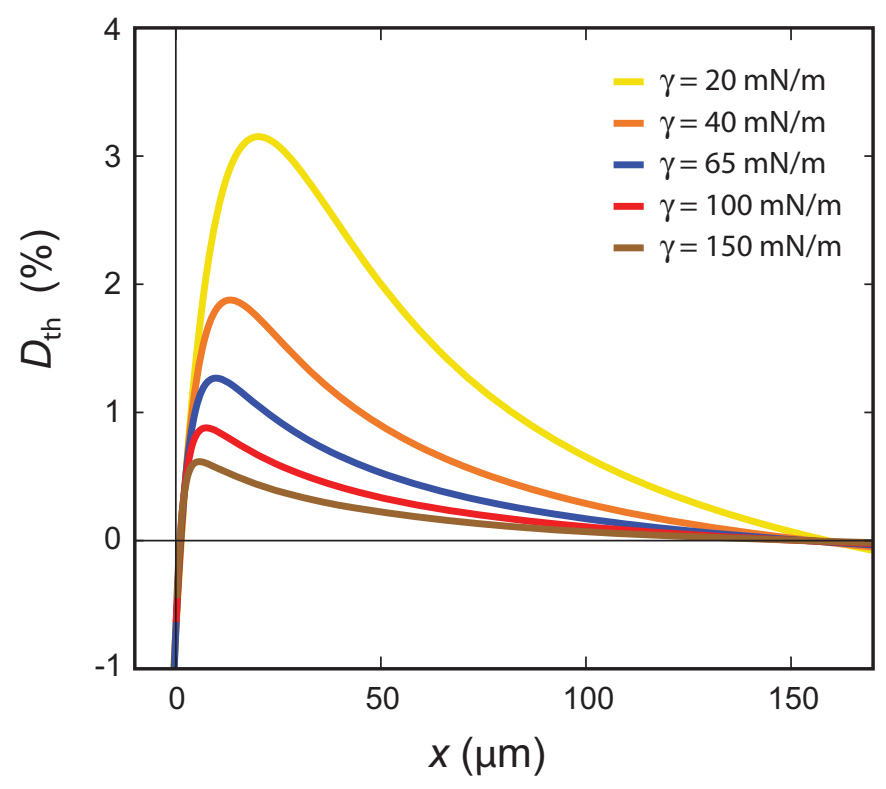

(a)

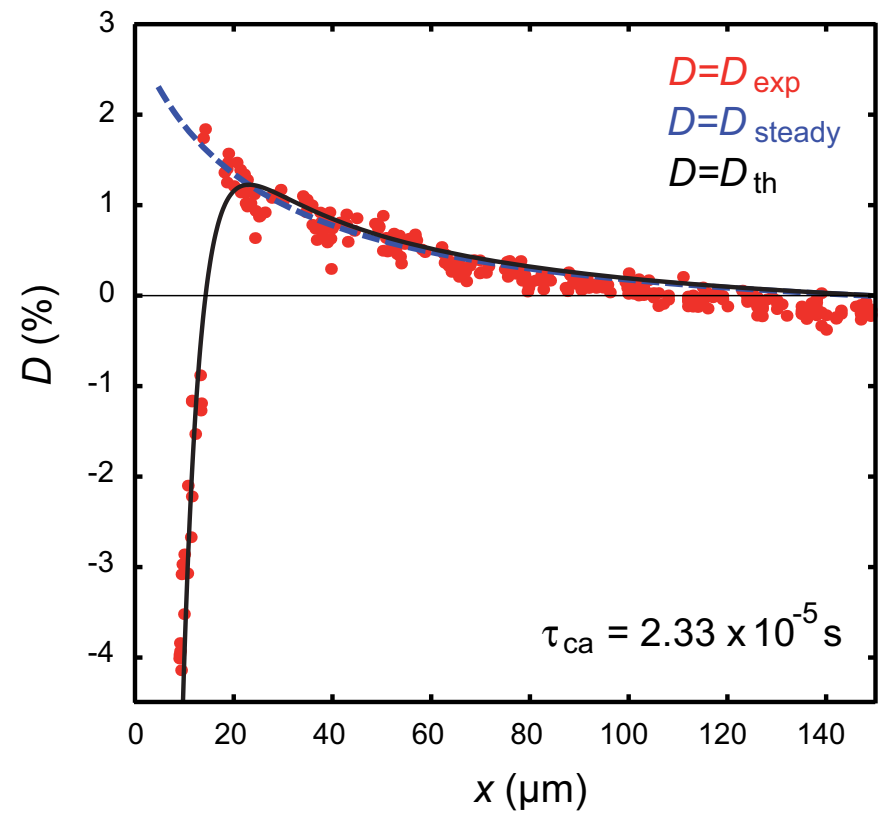

(b)

FIG. 4. (a) Calculated theoretical deformation $D_{\text {th }}$ as a function of droplet position $x$, for various values of the droplet-medium interfacial tension $\gamma$ as indicated and an arbitrary initial condition for illustration. $D_{\mathrm{th}}(x)$ is obtained by integrating Eq. (3) along the trajectory of a droplet advected by the flow, and invoking the simulated $D_{\text {steady }}(x)$ [Fig. 3(b)]. (b) Fit of the experimental data $D_{\exp }(x)$ (dots, collected for 28 droplets) to the calculated theoretical deformation $D_{\text {th }}(x)$, integrated with the first data point as an initial condition. The best-fit parameter $\tau_{\text {ca }}$ [see Eqs. (3) and (4)] is indicated. 


\section{Comparison with experimental data}

As it is not possible to produce stable microdroplets without surfactant, we stabilize them with poly(methacrylic acid) (PMAA) [21]. In contrast to common surfactant molecules, these polymer chains do not easily desorb and can stabilize droplets, even with a relatively low quantity of molecules adsorbed. In addition, the fluctuations of the interfacial tension due to local compression or dilatation of the surface during droplet deformation remain very low with these polymer chains. This is consistent with our model that assumes a homogeneous and constant droplet-medium interfacial tension during deformation. We note that, for more complex surface compositions, involving, e.g., surface viscoelasticity, Eq. (4) should be modified. This will be the object of another study.

The experimental data shown in Fig. 4(b) exhibits an evolution of the deformation in three main steps. First, the deformation is negative due to the initial confinement of the droplet in the narrow channel. Indeed, the latter is $40 \mu \mathrm{m}$ wide, while the droplet diameter ranges between 50 and $70 \mu \mathrm{m}$. The deformation then increases toward positive values, due to the diverging viscous flow which elongates the droplet in the $y$ direction. Finally, for larger values of $x$, when the droplet is further away from the diverging region, the deformation gradually relaxes to 0 (i.e., a spherical shape) due to surface tension.

The fitting of the experimental data, for 28 different droplets, to the theoretical prediction is shown as well in Fig. 4(b). The agreement is good, and the best-fit capillary time [see Eqs. (3) and (4)] is found to be $\tau_{\mathrm{ca}}=2.33 \times 10^{-5} \mathrm{~s}$. From this value, we deduce a droplet-medium interfacial tension $\gamma=35 \pm 3 \mathrm{mN} / \mathrm{m}$, which is in good agreement with what can be measured using a pendant-drop apparatus $(\gamma=40 \pm 1 \mathrm{mN} / \mathrm{m})$. Note that the droplet history, size, and viscous-shear conditions are very different between the two experiments, which could explain the small difference.

\section{CONCLUSION}

The transient deformation of a droplet in the extensional flow following a microfluidic constriction is a model problem connected to important applications, from encapsulated-drug delivery to cancercell detection [22]. Because of the absence of any symmetry in the associated flow and to the influence of the droplet itself on the latter, the simple approaches of the literature are not valid in general. Using finite-element simulations, we have shown that it is possible to predict the shear stress applied on the droplet and thus the resulting droplet deformation. Our model captures well our experimental data, performed with a large collection of polymer-stabilized oil microdroplets in water, which enables the robust statistical measurement of the droplet-medium interfacial tension. This model study opens the way toward precise in situ microrheology of capsules and cells, with more complex viscoelastic behaviors.

\section{ACKNOWLEDGMENTS}

This work was financially supported by ANR JCJC INTERPOL and AMX from Ecole Polytechnique. The authors thank Mamisoa Nomena and Samuel Poincloux for their valuable help and advice in this work. They also thank Oliver Bäumchen, Ingmar Polenz, Jean-Christophe Baret, Jan Vermant, Julien Dupré de Baubigny, Nadège Pantoustier, and Patrick Perrin for fruitful discussions. Finally, they acknowledge funding from the Global Station for Soft Matter, a project of Global Institution for Collaborative Research and Education at Hokkaido University.

\section{APPENDIX}

A MATLAB program enables us to process the large number of pictures. For each of them, the background is subtracted using a reference picture with no droplet, and a threshold is automatically set to detect the droplet. For every picture containing at least one entire droplet, the position, the mean radius, and the deformation of the droplet are calculated. The droplet position $(x, y)$ is the mean position of all the pixels forming the droplet. 
The mean radius $r$, defined as the radius of the droplet at rest, is calculated with the two semiaxes $a$ and $b$, in the directions $x$ and $y$ respectively [see Fig. 1(c)]. For a small deformation, any deviation from the spherical shape is indeed an ellipsoid at second order in deformation, as noted by Cox [23] and Rallison [17]. Moreover, due to the flow orientation, the axes of the droplet are along $x$ and $y$. By volume conservation, and noting $c$ the semiaxis in the $z$ direction, one gets

$$
\frac{4}{3} \pi r^{3}=\frac{4}{3} \pi a b c
$$

We assume that when the shear flow elongates the capsule in one direction, the $z$ direction is compressed by the same amount as the other compressed direction; i.e., $c$ is equal to the smallest semiaxis among $a$ and $b$. Consequently, using Eq. (A1), one has

$$
r=[a b \min (a, b)]^{1 / 3} .
$$

The deformation $D$ is then defined from the semiaxes $a$ and $b$, as follows:

$$
D=\frac{b-a}{b+a}
$$

As suggested by Martin et al. [20], in the ellipsoidal approximation justified by Cox [23], it is in fact more precise to calculate the deformation through the moments of inertia along the $x$ and the $y$ axes, respectively, $I_{x}$ and $I_{y}$ :

$$
D=\frac{\sqrt{I_{y}}-\sqrt{I_{x}}}{\sqrt{I_{y}}+\sqrt{I_{x}}},
$$

where

$$
I_{x}=\sum_{\text {pixels i }}\left(x_{i}-x\right)^{2},
$$

and

$$
I_{y}=\sum_{\text {pixels i }}\left(y_{i}-y\right)^{2}
$$

where $x_{i}$ and $y_{i}$ are the pixel coordinates [see Fig. 1(c)]. This is the method we employ in our study.

Consequently, the position and deformation of the droplet are known precisely for every picture containing a droplet. The time lapse between two consecutive pictures is $\Delta t=0.1 \mathrm{~ms}$ and, despite the high acquisition rate, we have only approximately six pictures per droplet due to the high flow rate. For every droplet $n$, the time $t_{n}$ is counted starting from the first picture where the droplet is recorded. Therefore, $t_{n}$ is always a multiple of $\Delta t$. The function $t_{n}(x)$ is fitted by a second-order polynomial function:

$$
t_{n}=a_{n} x^{2}+b_{n} x+c_{n}
$$

All the droplets are finally synchronized using a common time measurement $t_{\text {sync }}$, defined as $t_{\text {sync }}=t_{n}-c_{n}$, and characterized by $t_{\text {sync }}(x=0)=0$. The good droplet monodispersity and the high acquisition rate of the camera allow us to acquire enough data in a short period of time ( $2 \mathrm{~s})$, during which the flow and the thermodynamic conditions remain constant. The measurements for different droplets can therefore be concatenated to generate one single curve $D_{\text {exp }}(x)$ [see Fig. 4(b)]. 
[1] H. C. Shum, J. W. Kim, and D. A. Weitz, Microfluidic fabrication of monodisperse biocompatible and biodegradable polymersomes with controlled permeability, J. Am. Chem. Soc. 130, 9543 (2008).

[2] R. Seemann, M. Brinkmann, T. Pfohl, and S. Herminghaus, Droplet based microfluidics, Rep. Prog. Phys. 75, 16601 (2012).

[3] R. M. Parker, J. Zhang, Y. Zheng, R. J. Coulston, C. A. Smith, A. R. Salmon, Z. Yu, O. A. Scherman, and C. Abell, Electrostatically directed self-assembly of ultrathin supramolecular polymer microcapsules, Adv. Funct. Mater. 25, 4091 (2015).

[4] D. F. do Nascimento, L. R. Arriaga, M. Eggersdorfer, R. Ziblat, M. de Fatima, V. Marques, F. Reynaud, S. A. Koehler, and D. A. Weitz, Microfluidic fabrication of pluronic vesicles with controlled permeability, Langmuir 32, 5350 (2016).

[5] J. Wang, Y. Li, X. Wang, J. Wang, H. Tian, P. Zhao, Y. Tian, Y. Gu, L. Wang, and C. Wang, Droplet microfluidics for the production of microparticles and nanoparticles, Micromachines 8, 22 (2017).

[6] G. I. Taylor, The formation of emulsions in definable fields of flow, Proc. R. Soc. London, Ser. A 146, 501 (1934).

[7] J. S. Lee, R. Dylla-Spears, N. P. Teclemariam, and S. J. Muller, Microfluidic four-roll mill for all flow types, Appl. Phys. Lett. 90, 074103 (2007).

[8] J. Deschamps, V. Kantsler, E. Segre, and V. Steinberg, Dynamics of a vesicle in general flow. Proceed. Nat. Acad. Sci. USA 106, 11444 (2009).

[9] K. Xie, C. de Loubens, F. Dubreuil, D. Z. Gunes, M. Jaeger, and M. Leonetti, Interfacial rheological properties of self-assembling biopolymer microcapsules, Soft Matter 13, 6208 (2017).

[10] S. D. Hudson, J. T. Cabral, W. J. Goodrum, K. L. Beers, and E. J. Amis, Microfluidic interfacial tensiometry, Appl. Phys. Lett. 87, 081905 (2005).

[11] J. T. Cabral and S. D. Hudson, Microfluidic approach for rapid multicomponent interfacial tensiometry, Lab Chip 6, 427 (2006).

[12] Q. Brosseau, J. Vrignon, and J.-C. Baret, Microfluidic dynamic interfacial tensiometry ( $\mu$ DIT), Soft Matter 10, 3066 (2014).

[13] D. Barthes-Biesel and A. Acrivos, Deformation and burst of a liquid droplet freely suspended in a linear shear field, J. Fluid Mech. 61, 1 (1973).

[14] D. Bartolo, G. Degre, P. Nghe, and V. Studer, Microfluidic stickers, Lab Chip 8, 274 (2008).

[15] I. Polenz, Q. Brosseau, and J.-C. Baret, Monitoring reactive microencapsulation dynamics using microfluidics, Soft Matter 11, 2916 (2015).

[16] D. Barthes-Biesel and J. M. Rallison, The time-dependent deformation of a capsule freely suspended in a linear shear flow, J. Fluid Mech. 113, 251 (1981).

[17] J. M. Rallison, The deformation of small viscous drops and bubbles in shear flows, Annu. Rev. Fluid Mech. 16, 45 (1984).

[18] E. Lauga, M. P. Brenner, and H. A. Stone, Microfluidics: The no-slip boundary condition, In Springer Handbook of Experimental Fluid Mechanics (Springer, Berlin, 2005), Chap. 19, pp. 1219-1240.

[19] C. D. Eggleton, Y. P. Pawar, and K. J. Stebe, Insoluble surfactants on a drop in an extensional flow: A generalization of the stagnated surface limit to deforming interfaces, J. Fluid Mech. 385, 79 (1999).

[20] J. D. Martin and S. D. Hudson, Mass transfer and interfacial properties in two-phase microchannel flows, New J. Phys. 11, 115005 (2009).

[21] S. le Tirilly, C. Tregouet, M. Reyssat, S. Bone, C. Geffroy, G. G. Fuller, N. Pantoustier, P. Perrin, and C. Monteux, Interfacial rheology of hydrogen-bonded polymer multilayers assembled at liquid interfaces: Influence of anchoring energy and hydrophobic interactions, Langmuir 32, 6089 (2016).

[22] S. Byun, S. Son, D. Amodei, N. Cermak, J. Shaw, J. H. Kang, V. C. Hecht, M. Winslow, T. Jacks, P. Mallick, and S. R. Manalis, Characterizing deformability and surface friction of cancer cells, Proc. Natl. Acad. Sci. USA 110, 7580 (2013).

[23] R. G. Cox, The deformation of a drop in a general time-dependent fluid flow, J. Fluid Mech. 37, 601 (1969). 